\title{
Comparison of potassium and sodium binding in vivo and in agarose samples using TQTPPI pulse sequence
}

\author{
Victor D. Schepkin ${ }^{1}$, Andreas Neubauer ${ }^{2}$, Armin M. Nage $\left.\right|^{3,4}$ \\ Thomas F. Budinger ${ }^{5}$
}

\author{
${ }^{1}$ CIMAR, National High Magnetic Field Laboratory/FSU, Tallahassee, FL, USA \\ ${ }^{2}$ Computer Assisted Clinical Medicine/CBTM, Heidelberg University, Mannheim, Germany \\ ${ }^{3}$ German Cancer Research Center (DKFZ), Heidelberg, Germany \\ ${ }^{4}$ Institute of Radiology, University Hospital Erlangen, Erlangen, Germany \\ ${ }^{5}$ Lawrence Berkeley National Laboratory/UCB, Berkeley, CA, USA
}

Running title: TQTPPI detection of potassium and sodium

Address for correspondence:

Victor Schepkin, Ph.D.

Research Faculty II

Center for Interdisciplinary Magnetic Resonance

National High Magnetic Field Laboratory

1800 East Paul Dirac Drive

Tallahassee, FL 32310

Tel: 850-645-7357

Fax: 850-644-1366

Email: schepkin@magnet.fsu.edu

Manuscript word count: 5955 words 


\section{List of abbreviations:}

$\mathrm{TQ}$ - triple quantum

$S Q$ - single quantum

$M Q$ - multiple quantum

TPPI - time proportional phase increment

FT - Fourier transformation

$\mathrm{RF}$ - radio frequency

$\mathrm{TQ}^{\mathrm{A}}-\mathrm{TQ}$ MR peak area

$S Q^{A}-S Q M R$ peak area

$A_{S Q F}-S Q M R$ signal amplitude, fast relaxing component

$A_{S Q L}-S Q M R$ signal amplitude, slowly relaxing component

$A_{S Q}$ - total SQ MR signal amplitude, sum of the fast and slowly relaxing components $A_{T Q}-T Q M R$ signal amplitude

$T_{2 F}-T_{2}$ relaxation time, fast component

$T_{2 L}-T_{2}$ relaxation time, slow component

t-max - position of the TQ signal maximum

ns - number of steps 


\section{Abstract}

Potassium and sodium specific binding in vivo were explored at $21.1 \mathrm{~T}$ by triple quantum (TQ) magnetic resonance (MR) signals without filtration to achieve high sensitivities and precise quantifications. The pulse sequence used time proportional phase increments (TPPI). During simultaneous phase-time increments, it provided total single quantum (SQ) and TQ MR signals at single and triple quantum frequencies, respectively. The detection of both $T Q$ and $S Q$ signals was performed at identical experimental conditions and the resulting TQ signal equals $60 \pm 3 \%$ of the SQ signal when all ions experience sufficient time for binding. In a rat head in vivo the TQ percentage relative to $S Q$ for potassium is $41.5 \pm 3 \%$ and for sodium is $16.1 \pm 1 \%$. These percentages were compared to the matching values in agarose tissue model with MR relaxation times similar to those of mammalian brain tissue. The sodium TQ signal in agarose samples decreased in the presence of potassium, suggesting a competitive binding of potassium relative to sodium ions for the same binding sites. The TQTPPI signals correspond to almost two times more effective binding of potassium than sodium. In vivo, up to $\sim 69 \%$ of total potassium and $\sim 27 \%$ of total sodium can be regarded as bound or experiencing an association time in the range of several milliseconds. Experimental data analyses show that more than half of the in vivo total sodium TQ signal could be from extracellular space, which is an important factor for quantification of intracellular MR signals.

Key words: in vivo; rat; triple quantum NMR; potassium; sodium; binding; TQTPPI; 


\section{Introduction}

Potassium and sodium are major ions in vivo and their MR signals have great potential to convey valuable information about cell functioning (1-5). The difference in concentration of potassium and sodium between intracellular and extracellular sites is an important indicator of cellular energy metabolism (6-8) and the concentration gradients give rise to neuronal membrane potentials essential for nerve activity.

In addition to in vivo studies of the single quantum (SQ) signals from sodium in brain and heart, the triple quantum (TQ) MR signals from sodium and potassium have come under investigation (9-18) because of their potential to reveal changes in intracellular ion content without using contrast agents. For example, after calibration, the TQ filtered (TQF) sodium MR signal can measure changes in the intracellular sodium concentration in an isolated perfused rat heart without shift reagents (19).

Sodium and potassium nuclei have spin $S=3 / 2$, thus these ions interact with the surrounding electric field gradients of the macromolecules in vivo with small $(<200 \mathrm{~Hz})$ quadrupole interactions. The effect of such interactions on the signal cannot be observed as separate satellite MR peaks. However, there are exceptions as was detected for potassium in muscle (20), sodium in cartilage (21) and the excised bovine optic nerve (22). The fraction of such MR signals is very small in the rat head relative to the total MR signals of sodium or potassium and was not observed in our experiments. In vivo macromolecules provide many electro-negative interaction sites for sodium and potassium. These macromolecules are mainly proteins, nucleic acids and carbohydrates with exposed carboxyl groups $(-\mathrm{COOH})$, hydroxyl groups $(-\mathrm{COH})$ and phosphate groups $\left(\mathrm{PO}_{4}{ }^{3-}\right)$. All these groups are involved in interaction with the positively charged ions of potassium and sodium with binding times in the range of several milliseconds. These interactions result in the TQ MR signal.

A widespread and accurate use of $T Q$ signals is currently hindered by the low sensitivity of sodium and particular potassium NMR signals. Historically, the application of TQF pulse sequences was the primary way to detect TQ signals. TQF detection is usually performed by the pulse sequence $90-\tau-90-\delta-90$ (Fig. 1) using a specially selected phase cycling. The TQF pulse sequence interval " $\tau$ " is usually maximized for the most prevailing time of binding from a wide range of ion binding times existing in- 
vivo; a typical value for interval " $\tau$ " is $2-6 \mathrm{~ms}$. The TQF signal in-vivo, detected by such pulse sequences is only a small fraction of the total single quantum (SQ) signal.
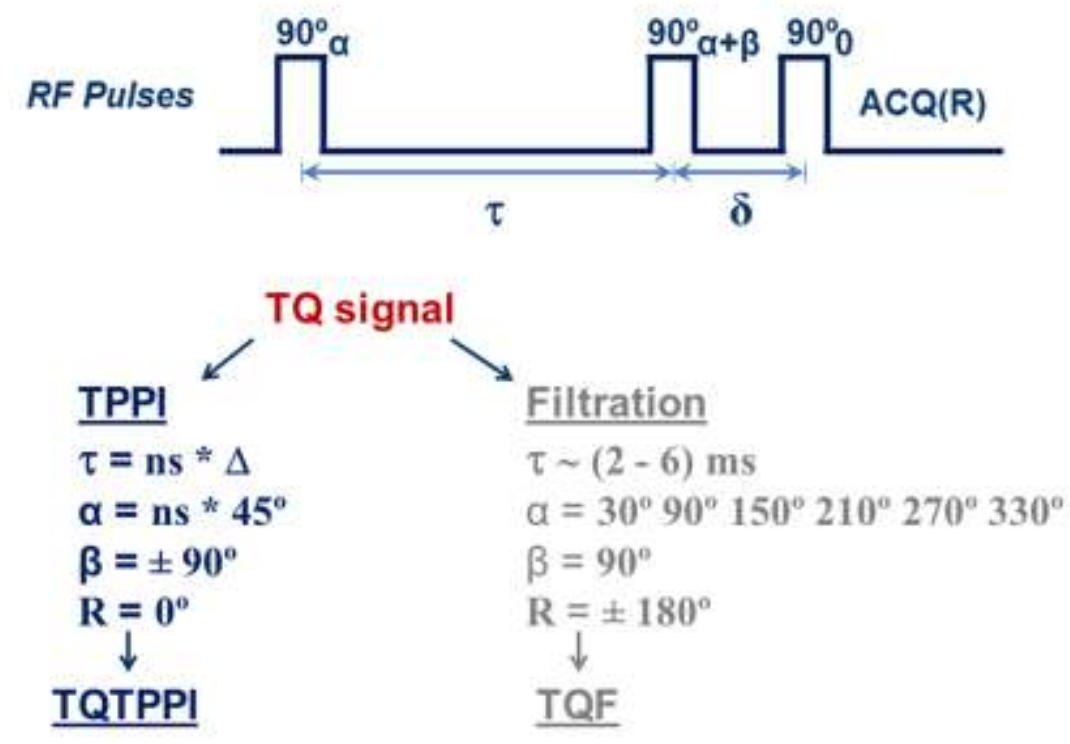

Fig. 1. Comparison of the TQTPPI and TQF pulse sequences. The TQTPPI pulse sequence has simultaneous increments of interval $\tau=n s^{*} \Delta$ and RF phase $\alpha=n s^{*} 45^{\circ}$ (ns is a number of steps). The RF phase $\beta$ has values of $+90^{\circ}$ and $-90^{\circ}$ for each value of $\tau$ and $\alpha$. Both results are added together to suppress a double quantum signal. The receiver phase $R$ remains unchanged. In the TQF pulse sequence, the interval " $\tau$ " has a fixed value in the range of $2-6$ $m s$. The phase $\alpha$ changes throughout the cycle of 6 steps, while the receiver phase alternates $\pm 180^{\circ}$ with each RF phase step. A sum of all signals gives the TQF signal. A 180 degree pulse $\left(180_{\alpha+90^{\circ}}\right)$ at the middle of interval " $\tau$ " is not shown.

A dramatic improvement in the efficiency and accuracy of the TQ signal detection can be achieved by avoiding filtration using the TQTPPI method (Fig. 1). In comparison to a typical TPPI pulse sequence $(23,24)$, where the phase increment is $90^{\circ}$, the largest phase increment to detect the TQ signal is $45^{\circ}$. Smaller phase steps are also acceptable, for example $30^{\circ}$. The TQTPPI has the same efficiency for a wide range of ion bindings. The TQ MR signals are acquired concurrently with $S Q$ peak. For example, during the $45^{\circ}$ phase increment a full cycle (period of $360^{\circ}$ ) is covered by 8 phase steps. Thus, after Fourier transform (FT) in the second dimension, the SQ peak 
can be found at $1 /\left(8^{*} \Delta \tau\right)$, which is well separated in the frequency domain from the TQ signal at $3 /\left(8^{*} \Delta \tau\right)$.

The TQTPPI pulse sequence for sodium was previously shown in the test samples $(25,26)$ and in vivo (27). The current study is devoted to the application and analysis of the TQTPPI pulse sequence for potassium and sodium in a rat head at 21.1 T. The results were compared with the corresponding data from conventional TQF experiments. Additionally the same experiments were conducted in a model system of brain tissue consisting of $7.5 \%$ agarose. This concentration of agarose gives the MR relaxation parameters close to the in vivo results (28). As will be shown, the model system was able to demonstrate a stronger and competitive binding of potassium relative to sodium for the same binding places.

\section{Methods}

The experiments were performed on a 21.1T magnet using Bruker MRI Avance III console (PV 5.1) and a $64 \mathrm{~mm}$ gradient coil (RR, Inc.). Volume MRI coils for potassium $(41.8 \mathrm{MHz})$ and sodium $\left({ }^{23} \mathrm{Na}, 238 \mathrm{MHz}\right)$ were approximately the same size with ID/L = 33/54 mm. A double tuned $\mathrm{Na} / \mathrm{H}$ RF coil was described previously (29). The potassium coil was a single tuned RF coil of Alderman-Grant design (28).

A commonly used TQ filtering pulse sequence $90^{\circ}{ }_{\alpha}-\tau-90^{\circ}{ }_{\alpha+\beta}-\delta-90{ }^{\circ}$ was modified so that the phase " $\alpha$ " was incremented by $45^{\circ} \quad\left(\alpha=n s^{*} 45^{\circ}\right)$, at each step ns when the time delay $\left(\tau=n s^{*} \Delta\right)$ was incremented (Fig. 1). Before incrementing $\tau$ the phase " $\beta$ " was alternated $\left( \pm 90^{\circ}\right)$ and the results were added to suppress the double quantum (DQ) signal. The interval $\delta$ is usually selected to be the minimum allowed by the MR scanner hardware $(\sim 100 \mu \mathrm{s})$. The time increment $\Delta$ in TQTPPI pulse sequence was $100-300 \mu \mathrm{s}$, the typical durations of the $90^{\circ}$ pulse for sodium was $120 \mu \mathrm{s}$, and 140 $\mu$ s for potassium. In both pulse sequences, TQTPPI and TQF, a $180^{\circ}{ }_{\alpha+90}$ pulse was used in the middle of " $\tau$ " interval to compensate for the inhomogeneity of the magnetic field.

Free induction decays were acquired with "np" complex points, for potassium np $=2048$, and for sodium $n p=4092$. The number of increments "ns" was selected in the range 128 - 1024. The large numbers of steps $(>128)$ were selected to increase the 
accuracy of the spectra in the second dimension in some experiments. The spectral width was $25 \mathrm{KHz}$ for potassium and sodium. The number of accumulations NA and repetition time TR were for potassium NA $=16, T R=200 \mathrm{~ms}$, for sodium NA $=1$ and TR $=300 \mathrm{~ms}$. The FID signals were phased and Fourier transformed $(F T)$ in the first dimension. Then "ns" data points at the maximum of the central peaks of these spectra were selected (at the position of $(n p / 2+1)$ ). These points were used for a nonlinear fit in the time domain to derive amplitudes of the $S Q$ and $T Q$ signals. The same data were also FT transformed in the second dimension using the TPPI mode in which the phased real part was accompanied by zero filling for the omitted imaginary points. The area of the $S Q$ signal and both positive and negative parts of the TQ peak areas were calculated. The SQ peak was normalized to $100 \%$. Pre-processing of the above and other data was performed using MatNMR software v.3.9.94 (30). All results of the time domain fit and spectral peak areas are presented as mean \pm standard deviation.

The pulse sequence was first tested using model samples containing agarose gel $5 \%$ and $7.5 \%$ with added $\mathrm{NaCl}$ or $\mathrm{KCl}$ at concentration of $154 \mathrm{mM}$. The agarose (Carl Roth, Karlsruhe, Germany) was dissolved in the above solutions and heated up to $90^{\circ} \mathrm{C}$ with stirring. All model samples were placed in a plastic cylindrical container (diameter $=27 \mathrm{~mm}$ and length $=60 \mathrm{~mm}$; volume $=25 \mathrm{~mL}$..$A$ competition between $\mathrm{K}^{+}$and $\mathrm{Na}^{+}$ ions was observed when both $\mathrm{NaCl}(154 \mathrm{mM})$ and $\mathrm{KCl}(154 \mathrm{mM})$ were added to the agarose samples at the same time.

The TQTPPI potassium MRI signals were also acquired from polycrystalline potassium chloride $(\mathrm{KCl})$. The results served as a reference emulating a $100 \%$ binding of potassium. In this specific case of a solid sample, a 180 degree pulse at the middle of " $\tau$ " interval was not used.

The TQTPPI/TQF pulse sequences were applied to detect potassium and sodium TQ signals in five male Fisher 344 rats (weight $\sim 200 \mathrm{~g}$ ). The animals were anesthetized by breathing a $1.5 \% \mathrm{v} / \mathrm{v}$ isoflurane/air gas mixture. The rat head was reproducibly positioned in the RF coil to within $\pm 1 \mathrm{~mm}$ using a bite bar (29). The TQ signal maximum amplitude and its position t-max were calculated using a time fit of the TQ bi-exponential decay.

Animal experiments were conducted according to the protocols approved by the Florida State University ACUC. 


\section{Results}

\section{Comparison of potassium and sodium TQTPPI and TQF MR signals}

One way to process the TQTPPI data is to perform a non-linear fit of the total TQTPPI signal (Fig. 2) using the fitting function (31):

$Y=A_{S Q L} \sin (\omega t) \exp \left(-t / T_{2 L}\right)+A_{S Q F} \sin (\omega t) \exp \left(-t / T_{2 F}\right)+A_{T Q} \sin (3 \omega t)\left(\exp \left(-t / T_{2 L}\right)-\exp \left(-t / T_{2 F}\right)\right)+D C$

- where $\mathrm{Y}$ - amplitude of the fid signal, $\mathrm{t}$ - time, $\omega$ - frequency of the phase modulation $=$ $1 /\left(8^{*} \Delta\right), \Delta$ - time step increment, $D C$ is a base line shift, containing imperfect settings of the RF pulse durations and the accuracy of the phase corrections.

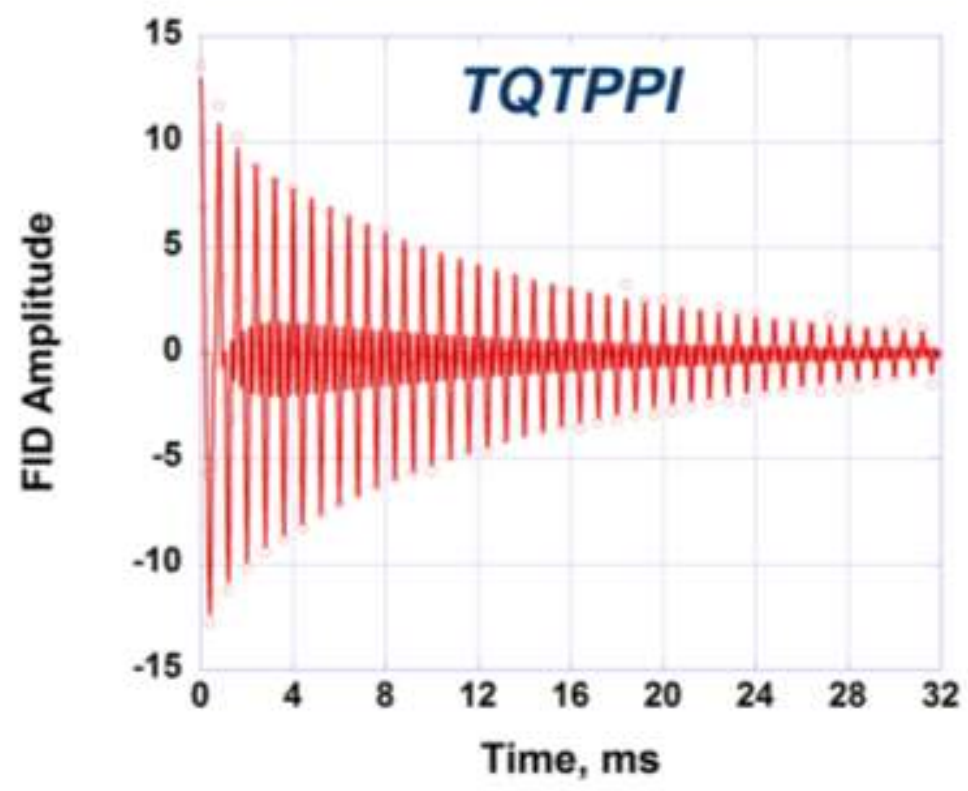

Fig. 2. Time course of potassium TQTPPI MR magnetization from a rat head in the second dimension. Note that the TQ MR signal of potassium appears early near the base line and slowly decays thereafter, during decay of the strong SQ potassium signal

The parameters of the time domain fit of the TQTPPI signals from the rat head as well as a corresponding TQF results are presented in Table 1. It is important to note that the single quantum signal has two exponential relaxation components (Fig. 3). 
Table 1. In vivo potassium and sodium TQ MR signal parameters in the rat head. The MR signal amplitudes are presented in percent relative to the total SQ signal. Note a less efficient sodium binding and the similarity of the TQTPPI and TQF parameters.

\begin{tabular}{|c|c|c|c|c|}
\hline $\begin{array}{c}\mathrm{TQ} \\
\text { parameters }\end{array}$ & $\begin{array}{c}\text { Potassium } \\
\mathrm{TQTPPI}\end{array}$ & $\begin{array}{c}\text { Potassium } \\
\mathrm{TQF}\end{array}$ & $\begin{array}{c}\text { Sodium } \\
\text { TQTPPI }\end{array}$ & $\begin{array}{c}\text { Sodium } \\
\text { TQF }\end{array}$ \\
\hline $\mathrm{TQ}^{\mathrm{A}} / \mathrm{SQ}^{\mathrm{A}}, \%$ & $41.5 \pm 3$ & & $16.1 \pm 1$ & \\
\hline $\mathrm{A}_{\mathrm{TQ}} / \mathrm{A}_{\mathrm{SQ}}, \%$ & $35.7 \pm 2.8$ & & $15.4 \pm 1$ & \\
\hline $\mathrm{A}_{\mathrm{SQF}} / \mathrm{A}_{\mathrm{SQ}}, \%$ & $55.3 \pm 0.9$ & & $41.6 \pm 0.9$ & \\
\hline $\mathrm{T}_{2 \mathrm{~L}}, \mathrm{~ms}$ & $13.3 \pm 0.4$ & $13.8 \pm 0.8$ & $30.6 \pm 2$ & $28.9 \pm 1$ \\
\hline $\mathrm{T}_{2 \mathrm{~F}}, \mathrm{~ms}$ & $0.79 \pm 0.03$ & $0.85 \pm 0.14$ & $2.3 \pm 0.2$ & $2.2 \pm 0.5$ \\
\hline $\mathrm{t}-\mathrm{max}, \mathrm{ms}$ & $2.37 \pm 0.07$ & $2.5 \pm 0.3$ & $6.5 \pm 0.6$ & $6.0 \pm 0.9$ \\
\hline
\end{tabular}

- $T Q^{A} / S Q^{A}$ - ratio for the $T Q$ peak area to the $S Q$ peak area in the TQTPPI spectrum. $A_{S Q}$ - total $S Q$ signal amplitude, $A_{S Q}=A_{S Q L}+A_{S Q F} ; A_{T Q}-T Q$ signal amplitude $\left(A_{S Q L}, A_{S Q F}\right.$ and $A_{T Q}$ are from TQTPPI time domain fit, formula (3)). $T_{2 L}$ and $T_{2 F}$ are slow and fast components of the biexponential relaxation of the $S Q$ and $T Q$ signals, $t$-max is a time position of the $T Q$ signal maximum. 
$S Q$

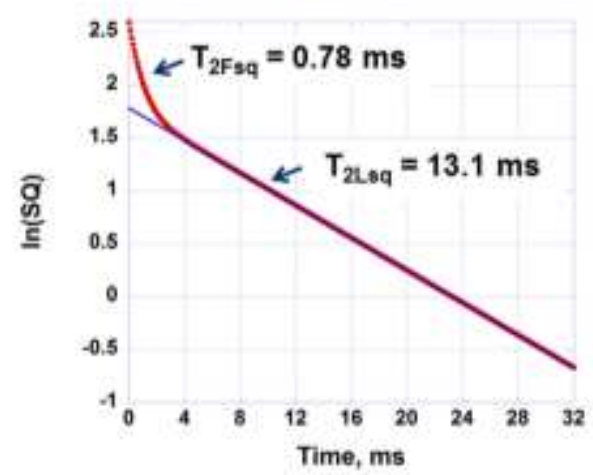

$T Q$

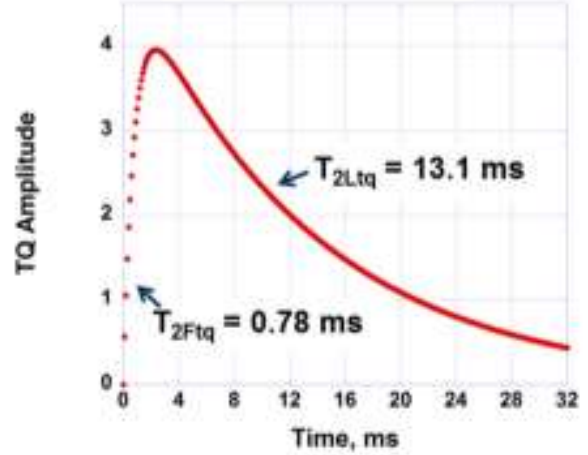

Fig. 3. The results of non-linear fit in the time domain of potassium TQTPPI magnetization from the rat head (from Fig. 2). Note the two-exponential decay for the $S Q$ signal, which represents a total MR potassium signal from a rat head.

The other way to process the TQTPPI data is to perform Fourier transformation in the second dimension of the TQTPPI data. The results are demonstrated for potassium and sodium in a normal rat head from in vivo studies (Fig. 4). The sodium SQ peak and the TQ peak are well separated having frequencies of $1.25 \mathrm{kHz}$ or $1 /\left(8^{*} \Delta\right)$ and $3.75 \mathrm{kHz}$ or $3 /\left(8^{*} \Delta\right)$ respectively. The TQ peak areas relative to the corresponding $\mathrm{SQ}$ peak areas $\left(\mathrm{TQ}^{\mathrm{A}} / \mathrm{SQ}^{\mathrm{A}}\right)$ were $41.5 \pm 3 \%$ for potassium and $16.1 \pm 1 \%$ for sodium. 


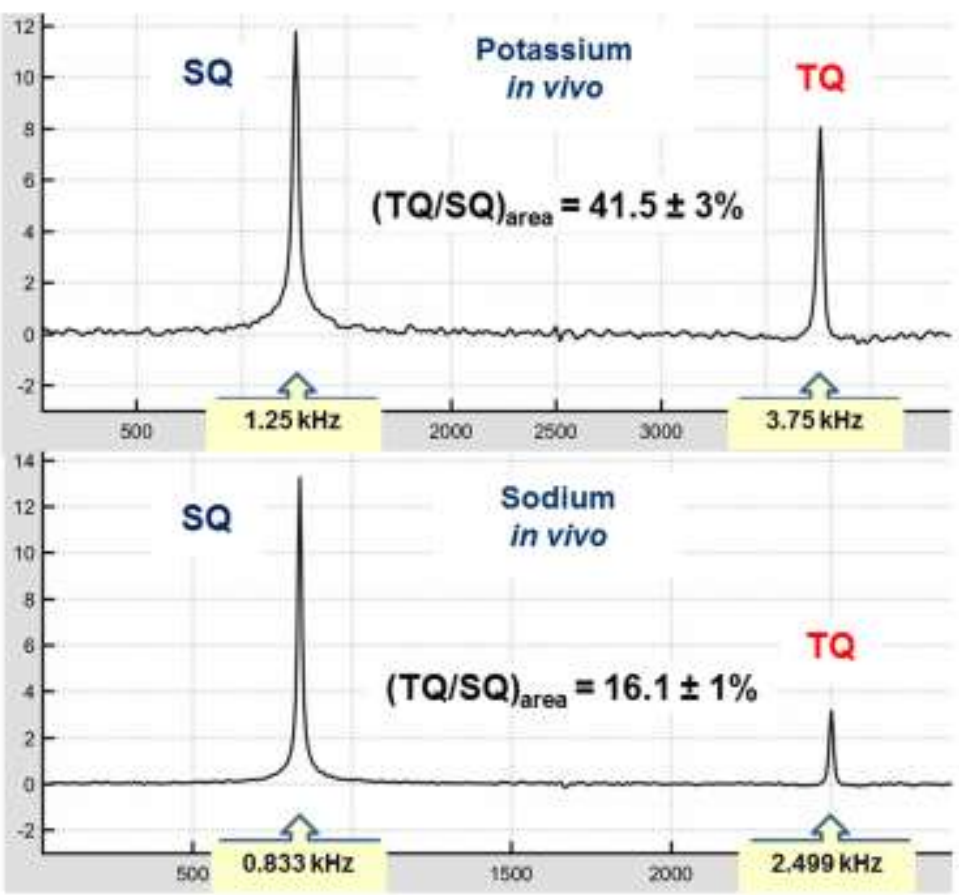

Fig. 4. TQTPPI spectra for potassium $(\Delta=0.1 \mathrm{~ms}, \mathrm{~ns}=320$, scan time $=30 \mathrm{~min})$ and sodium $(\Delta=0.15 \mathrm{~ms}, \mathrm{~ns}=760$, scan time $=7 \mathrm{~min})$ in the rat head at $21.1 \mathrm{~T}$. The percentages $\left(T Q^{A} / S Q^{A}\right)$ peak area for potassium was $41.5 \pm 3 \%$, and for sodium $16.1 \pm 1 \%$.

It is important to note that $\mathrm{SQ} M R$ signal represents a total MR signal, that is, both the bound and free ions of potassium and sodium. In contrast, the TQ signal represents only specifically bound ions.

For comparison, in the saline solution $(0.9 \% \mathrm{NaCl})$, the TQTPPI pulse sequence does not give a sodium TQ signal. The base line shows only noise level signals at the expected place for the TQ signal. Another example of the absence of a TQ signal is during sodium binding to glycerol. No TQ signal was detected despite the fact that binding of sodium to the glycerol molecule can shorten sodium MR relaxation times $T_{1}$ and $\mathrm{T}_{2}$ to about $8 \mathrm{~ms}$.

For quantification of the level of binding, solid polycrystalline $\mathrm{KCl}$ was used, because all potassium ions are in a bound state. The defects in the crystals are usually enough to produce small quadrupolar interactions at the position of potassium, which are fixed due to the limited mobility of potassium in the crystal. The TQTPPI spectrum gives the TQ peak area of $59.9 \pm 3 \%(n=6)$ relative to the SQ peak area (Fig. 5). This 
percentage is very close to a theoretical expectation for the intensity of the satellite transitions $1 / 2<->3 / 2$ of $60 \%(32,33)$.

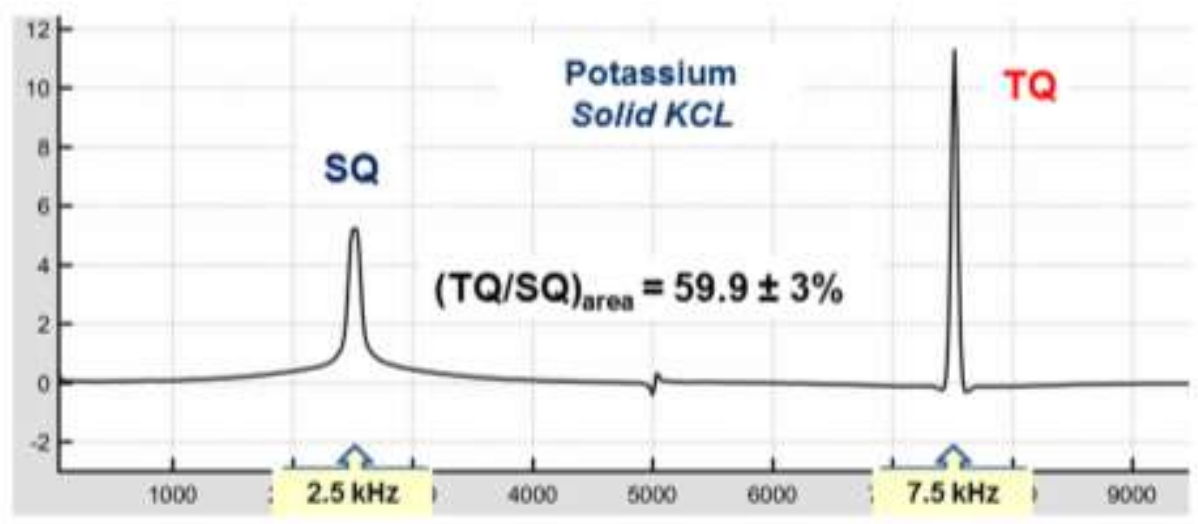

Fig. 5. Potassium TQTPPI spectrum from polycrystalline $K C L$ serves as a reference for the one hundred percent bound potassium system with $\left(T Q^{A} / S Q^{A}\right)$ peak area's ratio of $59.9 \pm 3 \%(\Delta$ $=0.05 \mathrm{~ms}, \mathrm{~ns}=400, \mathrm{TR}=30 \mathrm{sec}$, scan time $=6.5 \mathrm{~h}$ ). Thus, the TQ signal in the TQTPPI pulse sequence represents the intensity of the MR quadrupole satellite transitions.

The comparison of potassium and sodium binding was also performed in the test samples containing 5\% and 7.5\% agarose with $154 \mathrm{mM} \mathrm{KCl}$ or $154 \mathrm{mM} \mathrm{NaCl}$ (Table 2). The percentages of the peak areas of TQ signal to $S Q$ signal for potassium alone was $33.5 \pm 0.1 \%(n=3)$ in $5 \%$ agarose and increasing to $45.8 \pm 1 \%(n=3)$ in $7.5 \%$ agarose. The corresponding value for sodium were $25.7 \pm 0.7 \%(n=4)$ and $27.3 \pm 0.3 \%(n=4)$. The other parameters of the TQTPPI time domain fit are presented in Table 2.

Table 2. TQTPPI detection of the competitive potassium binding over sodium for the same binding places in 5\% (A) and $7.5 \%$ agarose $(B)$. MR signal amplitudes are presented in percent relative to the total $S Q$ signal. Note a decrease of sodium TQ signal in the presence of potassium in both cases: 
Table 2A. TQTPPI, Agarose 5\%

\begin{tabular}{|c|c|c|}
\hline Potassium & $\mathrm{KCl}(154 \mathrm{mM})$ & $\mathrm{KCl}(154 \mathrm{mM})+\mathrm{NaCl}(154 \mathrm{mM})$ \\
\hline$\left(\mathrm{TQ}^{\mathrm{A}} / \mathrm{SQ}^{\mathrm{A}}\right)_{\mathrm{K}}, \%$ & $33.5 \pm 0.1$ & $33.9 \pm 0.9$ \\
\hline$\left(\mathrm{A}_{\mathrm{TQ}} / \mathrm{A}_{\mathrm{SQ}}\right)_{\mathrm{K}}, \%$ & $32.5 \pm 0.3$ & $33.3 \pm 0.6$ \\
\hline$\left(A_{S Q F} / A_{S Q}\right)_{K}, \%$ & $58.9 \pm 0.4$ & $58.3 \pm 0.2$ \\
\hline $\mathrm{T}_{2 \mathrm{LK}}, \mathrm{ms}$ & $25.3 \pm 0.4$ & $23.8 \pm 1$ \\
\hline $\mathrm{T}_{2 \mathrm{FK}}, \mathrm{ms}$ & $1.96 \pm 0.1$ & $1.8 \pm 0.3$ \\
\hline$t-\max _{k}, \mathrm{~ms}$ & $5.4 \pm 0.1$ & $5.0 \pm 0.3$ \\
\hline Sodium & $\mathrm{NaCl}(154 \mathrm{mM})$ & $\mathrm{NaCl}(154 \mathrm{mM})+\mathrm{KCl}(154 \mathrm{mM})$ \\
\hline$\left(\mathrm{TQ}^{\mathrm{A}} / \mathrm{SQ}^{\mathrm{A}}\right)_{\mathrm{Na}}, \%$ & $25.7 \pm 0.7$ & $20.3 \pm 0.7$ * \\
\hline$\left(\mathrm{A}_{T Q} / \mathrm{A}_{\mathrm{SQ}}\right)_{\mathrm{Na}}, \%$ & $25.3 \pm 0.6$ & $21.7 \pm 0.7 *$ \\
\hline$\left(A_{S Q F} / A_{S Q}\right)_{\mathrm{Na}}, \%$ & $61 \pm 0.5$ & $60.2 \pm 1$ \\
\hline $\mathrm{T}_{2 \mathrm{~L} \mathrm{Na}}, \mathrm{ms}$ & $42.1 \pm 0.9$ & $43.4 \pm 1.7$ \\
\hline $\mathrm{T}_{2 \mathrm{~F} \mathrm{Na}}, \mathrm{ms}$ & $5.0 \pm 0.1$ & $6.6 \pm 0.4$ * \\
\hline$t-m_{N a x}, m s$ & $12.2 \pm 0.2$ & $14.7 \pm 0.8$ * \\
\hline
\end{tabular}


Table 2B. TQTPPI, Agarose $7.5 \%$

\begin{tabular}{|c|c|c|}
\hline Potassium & $\mathrm{KCl}(154 \mathrm{mM})$ & $\mathrm{KCl}(154 \mathrm{mM})+\mathrm{NaCl}(154 \mathrm{mM})$ \\
\hline$\left(\mathrm{TQ}^{\mathrm{A}} / \mathrm{SQ}^{\mathrm{A}}\right)_{\mathrm{K}}, \%$ & $45.8 \pm 0.9$ & $47.4 \pm 1.3$ \\
\hline$\left(\mathrm{A}_{\mathrm{TQ}} / \mathrm{A}_{\mathrm{SQ}}\right)_{\mathrm{K}}, \%$ & $42.4 \pm 0.5$ & $44.5 \pm 1.5$ \\
\hline$\left(\mathrm{A}_{\mathrm{SQF}} / \mathrm{A}_{\mathrm{SQ}}\right)_{\mathrm{K}} \%$ & $57.8 \pm 0.9$ & $57.2 \pm 1.4$ \\
\hline $\mathrm{T}_{2 \mathrm{~L} K}, \mathrm{~ms}$ & $14.5 \pm 0.5$ & $13.5 \pm 1.3$ \\
\hline $\mathrm{T}_{2 \mathrm{~F} \mathrm{~K}}, \mathrm{~ms}$ & $1.2 \pm 0.1$ & $1.07 \pm 0.1$ \\
\hline $\mathrm{t}-\mathrm{max}_{\mathrm{K}}, \mathrm{ms}$ & $3.3 \pm 0.1$ & $2.9 \pm 0.1$ \\
\hline Sodium $^{\mathrm{N}}$ & $\mathrm{NaCl}(154 \mathrm{mM})$ & $\mathrm{NaCl}(154 \mathrm{mM})+\mathrm{KCl}(154 \mathrm{mM})$ \\
\hline$\left(\mathrm{TQ}^{\mathrm{A}} / \mathrm{SQ}^{\mathrm{A}}\right)_{\mathrm{Na}}, \%$ & $27.3 \pm 0.3$ & $22.3 \pm 0.7^{*}$ \\
\hline$\left(\mathrm{A}_{\mathrm{TQ}} / \mathrm{A}_{\mathrm{SQ}}\right)_{\mathrm{Na}}, \%$ & $25.0 \pm 0.4$ & $22.3 \pm 0.7{ }^{*}$ \\
\hline$\left(\mathrm{A}_{\mathrm{SQF}} / \mathrm{A}_{\mathrm{SQ}}\right)_{\mathrm{Na}}, \%$ & $61.1 \pm 0.5$ & $61.2 \pm 0.7$ \\
\hline $\mathrm{T}_{2 \mathrm{~L} \mathrm{Na}}, \mathrm{ms}$ & $36.4 \pm 2.5$ & $37.4 \pm 3.8$ \\
\hline $\mathrm{T}_{2 \mathrm{Fa}}, \mathrm{ms}$ & $3.9 \pm 0.2$ & $4.9 \pm 0.4{ }^{*}$ \\
\hline $\mathrm{t}-\mathrm{max}_{\mathrm{Na}}, \mathrm{ms}$ & $9.7 \pm 0.6$ & $11.4 \pm 1{ }^{*}$ \\
\hline
\end{tabular}

- $\left(T Q^{A} / S Q^{A}\right)_{K},\left(T Q^{A} / S Q^{A}\right)_{N a}$ - ratios for the $T Q$ peak area to the $S Q$ peak area in the TQTPPI spectrum for potassium and sodium signals respectively. $A_{S Q}$-total $S Q$ signal amplitude, $A_{S Q}=$ $A_{S Q L}+A_{S Q F} ; A_{T Q}-T Q$ signal amplitude $\left(A_{S Q L}, A_{S Q F}\right.$ and $A_{T Q}$ are from TQTPPI time domain fit, formula (3)). $T_{2 L}$ and $T_{2 F}$ are slow and fast components of the bi-exponential relaxation of the $S Q$ and $T Q$ signals, $t$-max is a time position of the TQ signal maximum. * - difference is statistically significant.

The process of competition between potassium and sodium was detected by the TQTPPI method when both $\mathrm{KCl}$ and $\mathrm{NaCl}$ were simultaneously present with the same concentration of $154 \mathrm{mM}$. At both concentrations of agarose, the TQ/SQ ratio for sodium decreased significantly in the presence of potassium (Table 2). For the agarose concentration of $5 \%$, the sodium TQ peak area signal decreased from $25.7 \pm 0.7 \%$ ( $n=$ 4) to $20.3 \pm 0.7 \%(n=6)$. In the samples having $7.5 \%$ agarose, the corresponding decrease of sodium TQ signals is from $27.3 \pm 0.3 \%(n=4)$ to $22.3 \pm 0.7 \%(n=3)$.

These changes correlate with an increase of the sodium fast relaxing component $T_{2 F ~ N a}$ in the presence of potassium from $5.0 \pm 0.1 \mathrm{~ms}(n=4)$ to $6.6 \pm 0.4 \mathrm{~ms}(n=6)$ for $5 \%$ agarose samples and from $3.9 \pm 0.2 \mathrm{~ms}(n=4)$ to $4.9 \pm 0.4 \mathrm{~ms}(n=3)$ for $7.5 \%$ 
agarose samples. The consistent increase is also observed for the position of sodium TQ signal maximum in the presence of potassium from $12.2 \pm 0.2 \mathrm{~ms}(\mathrm{n}=4)$ to $14.7 \pm$ $0.8 \mathrm{~ms}(\mathrm{n}=6)$ for $5 \%$ agarose samples. In the samples with $7.5 \%$ agarose the corresponding increase is from $9.7 \pm 0.6 \mathrm{~ms}(n=4)$ to $11.4 \pm 1 \mathrm{~ms}(n=3)$ (Table 2).

\section{Discussion}

The polycrystalline $\mathrm{KCl}$ was selected to serve as a reference system where all potassium ions are bound. The application of the TQTPPI pulse sequence (Fig. 5) demonstrates that the experimental TQ peak area is very close to the theoretically expected value of $60 \%$ of the SQ peak area $(32,33)$. Thus, empirically, the observed TQ signal in the TQTPPI pulse sequence represents the intensity of the quadrupole satellite transitions.

It is expected that small quadrupole interactions can decrease the intensity of the SQ peak signal (Fig. 5). In the TQTPPI sequence the same SQ signal is used to detect both $S Q$ and TQ signal in the second dimension. Thus, the ratio of the TQ/SQ signals will represent the intensity of the satellite transition with a good accuracy.

Using the above result for polycrystalline $\mathrm{KCl}$ (Fig. 5) as a model for $100 \%$ binding, we can estimate from Table 1 that in vivo in the rat head approximately $41.5 \times 100 / 60=69 \%$ of total potassium has long term binding. The corresponding data for sodium in vivo suggests that much less, approximately $27 \%$, of sodium has long term binding.

The large difference in the relative TQ signal intensity between potassium and sodium raises a question if this is relevant to the predominant intracellular location of potassium. In efforts to find the answer, the TQTPPI signals of potassium and sodium were compared in the test samples containing $5 \%$ and $7.5 \%$ agarose (Table 2). The results of experiments, first of all, demonstrate that potassium, at the same binding places, gives a larger TQ/SQ ratio than sodium. In $5 \%$ agarose the percentage of $\mathrm{TQ}$ peak area relative to the $S Q$ peak area for potassium $\left(\mathrm{TQ}^{\mathrm{A}} / \mathrm{SQ}^{\mathrm{A}}\right)_{\mathrm{K}}=33.5 \%$, while that for sodium $\left(\mathrm{TQ}^{\mathrm{A}} / \mathrm{SQ}^{\mathrm{A}}\right)_{\mathrm{Na}}=25.7 \%$. The results for $7.5 \%$ agarose show for potassium $\left(\mathrm{TQ}^{\mathrm{A}} / \mathrm{SQ}^{\mathrm{A}}\right)_{\mathrm{K}}=45.7 \%$ and for sodium $\left(\mathrm{TQ}^{\mathrm{A}} / \mathrm{SQ}^{\mathrm{A}}\right)_{\mathrm{Na}}=27.3 \%$. Thus, both results in vivo and in the agarose samples show that potassium has a stronger TQ signal than sodium. This indicates a stronger binding of potassium. 
TQTPPI experiments offer additional and more direct evidence that potassium has stronger binding. Potassium can compete with sodium for the binding places as observed when both potassium and sodium were added together (Table 2). The results demonstrate that there is practically no change in potassium binding in the presence of sodium. For example, in the presence of sodium or without it, the percentage of the TQ peak area relative to the $S Q$ peak area $\left(T^{A} / S Q^{A}\right)_{K}$ for potassium remains at the same level of $33.7 \%$ for the $5 \%$ agarose sample and at a level of $46.5 \%$ for the $7.5 \%$ agarose sample. However, the TQ signal of sodium is decreased in presence of potassium. The TQ peak area relative to SQ peak area for sodium $\left(\mathrm{TQ}^{\mathrm{A}} / \mathrm{SQ}^{\mathrm{A}}\right)_{\mathrm{Na}}$ decreased from $25.7 \pm 0.7 \%$ to $20.3 \pm 0.7 \%$ for the $5 \%$ agarose samples in the presence of potassium and from $27.3 \pm 0.3 \%$ to $22.3 \pm 0.7 \%$ for the $7.5 \%$ agarose. Thus, it appears that potassium binding competes with bound sodium. These results correlate with experiments of others on changes of sodium $T_{1}$ relaxation times in the presence of phosphatidylserine vesicles due to the partial displacement of sodium by potassium (34). The preference of potassium binding over sodium was also detected in rat liver cell microsomes (35).

It is important to note that there is a difference for potassium between the ratio of the peak areas $\left(T Q^{A} / S Q^{A}\right)$ and the ratio for the amplitudes $\left(A_{T Q} / A_{S Q}\right)$ obtained from the time domain fit (Eq. 3) of the results of TQTPPI pulse sequence (Table 1). However, there is almost no such difference of these ratios for sodium. These results suggest that the two exponential fit of the TQ signal is only a first order of approximation. It is working for sodium and approximately for potassium in $5 \%$ agarose samples (Table 2A) and for sodium in vivo (Table 1$)$. In all other cases the deviation between $\left(T^{A} / S^{A}{ }^{A}\right)$ and $\left(A_{T Q} / A_{S Q}\right)$ can be observed suggesting more than two or a distribution of the $T_{2}$ relaxation times for bound ions.

Another important observation is that the fast decaying components of the SQ signal $\left(A_{S Q F} / A_{S Q}\right)_{K}$ and $\left(A_{S Q F} / A_{S Q}\right)_{N a}$ in the TQTPPI pulse sequence are of $57-61 \%$ for agarose samples (Table 2). These values are very close to a theoretical expectation of $60 \%$ for the intensity of the MR satellite peaks during quadrupole interaction. However, as it can be seen in the Table 2, the TQ signals in both cases are much less than $60 \%$, the expected value if all of ions experience quadrupole interaction. Thus, a short component of the SQ signal includes both ions with long time binding, represented by 
TQ signal and by ions bound to small molecules which may not yield contribution to the TQ signal.

In vivo, in the rat head, the $A_{S Q F} / A_{S Q}$ values are much smaller for sodium; it is $41.6 \%$, and it is a little less for potassium at $55.3 \%$ (Table 1 ). A value below $60 \%$ could be an indication that in vivo, a large fraction of sodium and a small fraction of potassium are in a "free" state, i.e. not experiencing significant long time quadrupole interactions.

\section{Estimation of sodium intracellular and extracellular TQ signals}

Using the above results for potassium and sodium TQ signals one can estimate the TQ signals from in vivo intracellular and extracellular spaces. The intracellular volume is assumed to be 0.85 of total tissue volume and the sodium intracellular and extracellular concentrations are assumed to be $15 \mathrm{mM}$ and $140 \mathrm{mM}$, respectively, which is close to estimations of others (36). In this case intracellular sodium content would be $0.378 \mathrm{Na}_{\mathrm{T}}$, and extracellular sodium content is $0.622 \mathrm{Na}_{\mathrm{T}}$, where $\mathrm{Na}_{\mathrm{T}}$ is the total sodium content in tissue. The difference between sodium and potassium TQ signals can be taken from the TQTPPI results in samples with $7.5 \%$ agarose (Table 2). These samples have $T_{1}, T_{2} M R$ relaxation parameters very close to sodium and potassium in vivo (28). Thus, from Table 2 sodium has $47.4 / 22.3=2.1$ times less TQ signal than potassium. The intracellular potassium TQ signal can be with high accuracy presented by the measured total potassium $\left(\mathrm{TQ}^{\mathrm{A}} / \mathrm{SQ}^{\mathrm{A}}\right)_{\mathrm{K}}=41.5 \%$ (Table 1 ) since contribution from extracellular potassium is very small. Assuming the concentration of potassium are $140 \mathrm{mM}$ intracellular and $4 \mathrm{mM}$ extracellular with intracellular volume of $85 \%$, only $0.5 \%$ of total potassium content is in extracellular space. Thus, it can be estimated that for intracellular sodium $\mathrm{TQ}^{\mathrm{A}}{ }_{\mathrm{Nai}} / \mathrm{SQ}^{\mathrm{A}}{ }_{\mathrm{Nai}}=41.5 / 2.1=19.8 \%$. The total $\left(\mathrm{TQ}^{\mathrm{A}} / \mathrm{SQ}^{\mathrm{A}}\right)_{\mathrm{Na}}$ signal from the rat head is $16.1 \%$, which was derived from our in vivo experiments. This value has respective contributions from extracellular and intracellular compartments as shown in equation 2

$$
\left(\mathrm{TQ}^{\mathrm{A}} \mathrm{Nai}+\mathrm{TQ}^{\mathrm{A}}{ }_{\mathrm{Naex}}\right) / \mathrm{SQ}^{\mathrm{A}}{ }_{\mathrm{Na}}=16.1 \%
$$

From this equation one can get the following value for extracellular sodium $\left(\mathrm{TQ}^{\mathrm{A}}{ }_{\text {Naex }} / \mathrm{SQ}^{\mathrm{A}}\right.$ Naex $)=13.9 \%$. During calculation it was used that $S Q^{\mathrm{A}} \mathrm{Na}=\mathrm{SQ}^{\mathrm{A}} \mathrm{Nai}+$ 
$\mathrm{SQ}^{\mathrm{A}}{ }_{\text {Naex }}, \mathrm{SQ}^{\mathrm{A}}{ }_{\text {Nail }} / \mathrm{SQ}^{\mathrm{A}}{ }_{\text {Naex }}=0.378 / 0.622=0.608$. This indicates that $\mathrm{TQ}$ binding of sodium in intracellular space is stronger $\left(\mathrm{TQ}^{\mathrm{A}}{ }_{\mathrm{Na}} / \mathrm{SQ}^{\mathrm{A}}{ }_{\mathrm{Nai}}\right) /\left(\mathrm{TQ}^{\mathrm{A}}{ }_{\text {Naex }} / \mathrm{SQ}^{\mathrm{A}}{ }_{\text {Naex }}\right)=19.8 / 13.9=$ 1.4 .

Thus, the total $\mathrm{TQ}^{\mathrm{Na}}$ signal of sodium is composed of two parts:

$$
\begin{aligned}
& \mathrm{TQ}^{\mathrm{A}}{ }_{\mathrm{Na}} / \mathrm{SQ}^{\mathrm{A}}{ }_{\mathrm{Na}}=7.5 \% \\
& \mathrm{TQ}^{\mathrm{A}}{ }_{\mathrm{Naex}} / \mathrm{SQ}^{\mathrm{A}} \mathrm{Na}=8.6 \%
\end{aligned}
$$

All percent values here are given relative to the total sodium SQ signal. These results indicate that TQ signals in the rat head from the extracellular space could be $53 \%$ relative to the total TQ signal. This estimation is compatible with the results of sodium TQ signal quantification performed in the rat heart (19), where it was found that extracellular TQ signal in the rat heart model can be approximately stable and is in the range of $50-64 \%$ of the total TQ signal from the normal rat heart. If in the estimation the intracellular volume is taken as 0.8 of the total tissue volume (due to CSF contribution) and sodium intracellular concentration is $15 \mathrm{mM}$, the TQ signal coming from extracellular space could be up to $64 \%$ of the total TQ signal. Thus, one can see the significant contribution of extracellular interactions to the TQ signal which was also noticed by others $(37,38)$. It is an important factor for quantification of intracellular sodium using the TQ signal (19).

A short relaxation time is not necessary associated with or give evidence of $T Q$ related binding. Sodium in a solution of glycerol and saline $(1: 1, \mathrm{v} / \mathrm{v})$ has short relaxation times $T_{1}=8.04 \pm 0.06 \mathrm{~ms}$ and $T_{2}=7.54 \pm 0.04 \mathrm{~ms}$ at $21.1 \mathrm{~T}$ and room temperature. However, the tumbling time of glycerol molecule is very short and not long enough to create any detectable TQ signal (27). Such types of binding can be present in vivo, which decreases sodium and potassium relaxation times without detectable $T Q$ signals (32). Thus, the part of the fast $S Q \mathrm{~T}_{2}$ component in vivo may have this type of origin. Consequently, the amplitude of the SQ short relaxing component and the TQ signal can be different.

During in vivo investigations, changes in tissue such as $\mathrm{pH}$ or oxygen partial pressure can be expected to result in a new distribution of binding strength. For 
example, during cell death in a rat heart, a maximum TQ signal occurs at an inter-pulse delay shorter than that for a normal heart (39). The deviation from an optimum TQ signal detection can be significant and if not taken into consideration, may lead to a wrong quantification. The TQTPPI pulse sequence does not have this problem. The changes in binding during interventions will be reflected by changes in the shape of the TQ signal peak, while the integral of the peak will be proportional to the amount of bound sodium. There is no need to perform an optimization of the inter-pulse delay " $\tau$ ". A small tissue RF power deposition relative to the spin-lock version of the pulse sequence is another valuable benefit. No extra reference is needed as the SQ signal can serve as a reference. In conclusion, the ratio of $T Q / S Q$ signals is a very sensitive parameter for detection of minor changes in binding.

The TQTPPI and TQF methods have different time efficiency. For a proper evaluation of the relative efficiency, it is important to include all the steps needed to conduct the TQF experiment. For the TQF strategy, one must first find the value between the first and second RF pulses that gives the maximum TQ signal. Thus, we need to acquire a number of delays depending on the required accuracy. Additionally, a separate experiment is needed to acquire a SQ signal. In the TQTPPI pulse sequence all these stages are combined, and there is no need for any extra experiments. When the inter-pulse delay is approximately known, the number of steps in the TQTPPI sequence can be as low as 8 . In this case, TQTPPI still preserves its capability to separate the TQ signal. The actual difference in acquisition time between TQTPPI and the TQF pulse sequences may depend on the in vivo model we use in the TQ experiments. If the inter-pulse delay is known and we do not expect it to change during the experiments, the TQF method may take less time. However, due to filtration, the TQF technique has less sensitivity to detect small TQ signals.

It is important to note that in vivo the majority of the binding sites are the negatively charged carbonyl and carboxyl groups, while in agarose samples the binding sites are carboxyl groups. Thus, the agarose model is not a complete model representative of the brain. The validity of the agarose tissue model is based on the fact that the relaxation times of potassium and sodium in agarose samples match those from the in vivo rodent head study (28). Additionally, the observed large TQ MR signals of potassium relative to sodium in agarose samples are comparable to the results in 
vivo. Thus, we expect that the agarose model system can reflect the main features of the TQ MR signals of the in vivo environment.

Finally, in the current study, the in vivo the MR signals from the whole rat head were used. There were no other localizations, except for the RF coil dimensions. The observed MR signals were a sum of the potassium or sodium MR signals from a rat brain and the surrounding muscles. The results have a value as it shows the total signal we obtain before using any localization technique. The MR imaging using TQTPPI approach is under development now, which could help in the localization of the signals from different parts of the head.

\section{Conclusion}

Simultaneous detection of SQ and TQ signals using the TQTPPI method provides an efficient method for evaluation of MR signals for potassium and sodium bound in vivo to macromolecules. The TQTPPI pulse sequence avoids the need for a separate $S Q$ pulse sequence as it does not cancel the $S Q$ signal, which is the case for the conventional TQF. The bound potassium and sodium signals are detected as separate peaks at the triple quantum frequency relative to the $S Q$ signal. The experimental conditions for SQ and TQ signals are the same which provide a high accuracy of the measurements. In comparison to the TQF methods, TQTPPI detects bound ion signals optimally for a wide range of binding strengths, which is important for evaluating ion concentration studies associated with diseases or interventions. Furthermore, the changes in sodium bindings can be monitored with an internal SQ reference signal, which is a valuable feature for in vivo applications. The TQ signal and its changes can be detected more sensitively, as there is no extra noise due to the filtration procedure. The quantification of the TQTPPI signals showed almost two times more effective binding of potassium than sodium, as well as a competitive binding of potassium relative to sodium for the same binding site. In vivo studies of the rat head showed up to $\sim 69 \%$ of total potassium and $\sim 27 \%$ of total sodium can be regarded as long-term bound or experience an association of several milliseconds during binding. Analysis of the results support the model that sodium ions in the extracellular space can contribute significantly to the total TQ signal. This specific feature of the TQ 
signals is little recognized but must be considered for studies that involve quantification of intracellular ions.

\section{Acknowledgement}

The study was performed at the National High Magnetic Field Laboratory (Tallahassee) which is supported by National Science Foundation Cooperative Agreement No. DMR-1157490 and the State of Florida. The authors thank Lothar R. Schad, Gregory S. Boebinger, Lucio Frydman and Timothy A. Cross for their support of this project. Many thanks to Steven Lee Ranner, Jason A. Kitchen, Ashley K. Blue, Richard L. Desilets, Peter L. Gor'kov and William W. Brey for the valuable and prompt help with RF probes. The authors also appreciate technical support from Shannon Helsper for help in animal handling. The authors appreciate efforts of Jacco van Beek in creation of the MatNMR software. 


\section{References}

1. Yu SP, Canzoniero LM, Choi DW. Ion homeostasis and apoptosis. Current opinion in cell biology 2001;13(4):405-411.

2. Bortner CD, Cidlowski JA. Uncoupling cell shrinkage from apoptosis reveals that $\mathrm{Na}+$ influx is required for volume loss during programmed cell death. J Biol Chem 2003;278(40):39176-39184.

3. Thulborn K, Lui E, Guntin J, Jamil S, Sun Z, Claiborne TC, Atkinson IC. Quantitative sodium MRI of the human brain at $9.4 \mathrm{~T}$ provides assessment of tissue sodium concentration and cell volume fraction during normal aging. NMR Biomed 2016;29(2):137-143.

4. Schepkin VD. Sodium MRI of glioma in animal models at ultrahigh magnetic fields. NMR Biomed 2016;29(2):175-186.

5. Madelin G, Regatte RR. Biomedical applications of sodium MRI in vivo. J Magn Reson Imaging 2013;38(3):511-529.

6. Silver IA, Deas J, Erecinska M. Ion homeostasis in brain cells: differences in intracellular ion responses to energy limitation between cultured neurons and glial cells. Neuroscience 1997;78(2):589-601.

7. Erecinska M, Dagani F. Relationships between the neuronal sodium/potassium pump and energy metabolism. Effects of $\mathrm{K}+, \mathrm{Na}+$, and adenosine triphosphate in isolated brain synaptosomes. J Gen Physiol 1990;95(4):591-616.

8. Erecinska M, Dagani F, Nelson D, Deas J, Silver IA. Relations between intracellular ions and energy metabolism: a study with monensin in synaptosomes, neurons, and C6 glioma cells. J Neurosci 1991;11(8):2410-2421.

9. Duvvuri U, Leigh JS, Reddy R. Detection of residual quadrupolar interaction in the human breast in vivo using sodium-23 multiple quantum spectroscopy. J Magn Reson Imaging 1999;9(3):391394.

10. Reddy R, Bolinger L, Shinnar M, Noyszewski E, Leigh JS. Detection of residual quadrupolar interaction in human skeletal muscle and brain in vivo via multiple quantum filtered sodium NMR spectra. Magn Reson Med 1995;33(1):134-139.

11. Hancu I, Boada FE, Shen GX. Three-dimensional triple-quantum-filtered (23) Na imaging of in vivo human brain. Magn Reson Med 1999;42(6):1146-1154.

12. Torres AM, Philp DJ, Kemp-Harper R, Garvey C, Kuchel PW. Determination of Na+ binding parameters by relaxation analysis of selected $23 \mathrm{Na}$ NMR coherences: RNA, BSA and SDS. Magn Reson Chem 2005;43(3):217-224.

13. Colet JM, Bansal N, Malloy CR, Sherry AD. Multiple quantum filtered 23Na NMR spectroscopy of the isolated, perfused rat liver. Magn Reson Med 1999;41(6):1127-1135.

14. Mirkes C, Shajan G, Bause J, Buckenmaier K, Hoffmann J, Scheffler K. Triple-quantum-filtered sodium imaging at 9.4 Tesla. Magnet Reson Med 2016;75(3):1278-1289.

15. Fleysher L, Oesingmann N, Brown R, Sodickson DK, Wiggins GC, Inglese M. Noninvasive quantification of intracellular sodium in human brain using ultrahigh-field MRI. NMR Biomed 2013;26(1):9-19.

16. Petracca M, Vancea RO, Fleysher L, Jonkman LE, Oesingmann N, Inglese M. Brain intra- and extracellular sodium concentration in multiple sclerosis: a 7 T MRI study. Brain 2016;139:795806.

17. Benkhedah N, Bachert P, Nagel AM. Two-pulse biexponential-weighted 23Na imaging. J Magn Reson 2014;240:67-76.

18. Madelin G, Lee JS, Regatte RR, Jerschow A. Sodium MRI: methods and applications. Prog Nucl Magn Reson Spectrosc 2014;79:14-47.

19. Schepkin VD, Choy IO, Budinger TF, Obayashi DY, Taylor SE, DeCampli WM, Amartur SC, Young JN. Sodium TQF NMR and intracellular sodium in isolated crystalloid perfused rat heart. Magn Reson Med 1998;39(4):557-563. 
20. Rosler MB, Nagel AM, Umathum R, Bachert $P$, Benkhedah N. In vivo observation of quadrupolar splitting in (39) K magnetic resonance spectroscopy of human muscle tissue. NMR in biomedicine 2016;29(4):451-457.

21. Eliav U, Navon G. Sodium NMR/MRI for anisotropic systems. NMR in biomedicine 2016;29(2):144-152.

22. Eliav U, Xu X, Jerschow A, Navon G. Optic nerve: Separating compartments based on Na-23 TQF spectra and TQF-diffusion anisotropy. J Magn Reson 2013;231:61-65.

23. Marion D, Wuthrich K. Application of the phase sensitive two-dimensional correlated spectroscopy (COSY) for measurements of $\mathrm{H}-1-\mathrm{H}-1$ spin-spin coupling constats in proteins. . Biochem Biophys Res Commun 1983;113(3):967-974.

24. Ernst RR, Bodenhausen G, Wokaun A. Principles of nuclear magnetic resonance in one and two dimensions. Oxford Oxfordshire, New York: Clarendon Press; Oxford University Press; 1987. xxiv, 610 p. p.

25. Van der Maarel JRC. Thermal relaxation and coherence dynamics of spin 3/2. I. Static and fluctuating quadrupolar interactions in the multipole basis. . Concept Magn Reson A 2003;19A(2):97-116.

26. Van der Maarel JRC. Relaxation of spin quantum number $S=3 / 2$ under multiple-pulse quadrupolar echoes. . J Chem Phys 1991;94(7):4765-4775.

27. Schepkin VD, Gawlick U, Ross BD, Chenevert TL. Detection of triple quantum Na NMR in normal and tumored mouse brain. Proceedings, 11th Annual Meeting, ISMRM, p 39. Volume 11. Toronto, Canada; 2003. p 39.

28. Nagel AM, Umathum R, Rosler MB, Ladd ME, Litvak I, Gor'kov PL, Brey WW, Schepkin VD. (39) K and (23) Na relaxation times and MRI of rat head at 21.1 T. NMR Biomed 2016;29(6):759-766.

29. Gor'kov PL, Qian C, Beck BL, Clark M, Masad IS, Schepkin VD, Grant SC, Brey WW. A modular MRI probe for large rodent neuroimaging at $21.1 \mathrm{~T}$ (900 MHz). Proceeding, 17th Annual Meeting, ISMRM, p 2952. Honolulu, Hawai'i, USA; 2009.

30. van Beek JD. matNMR: A flexible toolbox for processing, analyzing and visualizing magnetic resonance data in Matlab((R)). J Magn Reson 2007;187(1):19-26.

31. Jaccard G, Wimperis S, Bodenhausen G. Multiple-Quantum Nmr-Spectroscopy of S=3/2 Spins in Isotropic-Phase - a New Probe for Multiexponential Relaxation. J Chem Phys 1986;85(11):62826293.

32. Rooney WD, Springer CS, Jr. A comprehensive approach to the analysis and interpretation of the resonances of spins 3/2 from living systems. NMR Biomed 1991;4(5):209-226.

33. Abragam A. The principles of nuclear magnetism. Oxford,: Clarendon Press; 1961. 599 p. p.

34. Kurland R, Newton C, Nir S, Papahadjopoulos D. Specificity of $\mathrm{Na}+$ binding to phosphatidylserine vesicles from Na-23 NMR relaxation study. . Biochim Biophys Acta 1979;551(1):137-147.

35. Sanui $\mathrm{H}, \mathrm{Pace} \mathrm{N}$. Sodium and potassium binding by rat liver cell microsomes. . Journal of General Physiology 1959;42(6):1325-1345.

36. Thulborn KR, Davis D, Adams H, Gindin T, Zhou J. Quantitative tissue sodium concentration mapping of the growth of focal cerebral tumors with sodium magnetic resonance imaging. Magn Reson Med 1999;41(2):351-359.

37. Knubovets T, Shinar H, Navon G. Quantification of the contribution of extracellular sodium to 23Na multiple-quantum-filtered NMR spectra of suspensions of human red blood cells. J Magn Reson 1998;131(1):92-96.

38. Tauskela JS, Dizon JM, Whang J, Katz J. Evaluation of multiple-quantum-filtered 23Na NMR in monitoring intracellular $\mathrm{Na}$ content in the isolated perfused rat heart in the absence of a chemical-shift reagent. J Magn Reson 1997;127(1):115-127.

39. Schepkin VD, Choy IO, Budinger TF. Sodium alterations in isolated rat heart during cardioplegic arrest. J Appl Physiol 1996;81(6):2696-2702. 
